\title{
Patient-derived solitary fibrous tumour xenografts predict high sensitivity to doxorubicin/dacarbazine combination confirmed in the clinic and highlight the potential effectiveness of trabectedin or eribulin against this tumour ${ }^{\text {मे }}$
}

S. Stacchiotti ${ }^{\text {a,* }}$, M. Saponara ${ }^{\text {a }}$, R. Frapolli ${ }^{\text {b }}$, M. Tortoreto ${ }^{\text {c }}$, D. Cominetti ${ }^{c}$, S. Provenzano ${ }^{\text {a }}$, T. Negri ${ }^{\mathrm{d}}$, G.P. Dagrada ${ }^{\mathrm{d}}$, A. Gronchi ${ }^{\mathrm{e}}$, C. Colombo ${ }^{\mathrm{e}}$, B. Vincenzi ${ }^{\mathrm{f}}$, G. Badalamenti ${ }^{\mathrm{g}}$, V. Zuco ${ }^{\mathrm{c}}$, S.L. Renne ${ }^{\mathrm{h}}$, P. Collini ${ }^{h}$, C. Morosi ${ }^{i}$, A.P. Dei Tos ${ }^{j}$, E. Bello ${ }^{b}$, S. Pilotti ${ }^{d}$, P.G. Casali ${ }^{\mathrm{a}, \mathrm{k}}$, M. D'Incalci ${ }^{\mathrm{b}}$, N. Zaffaroni ${ }^{\mathrm{c}}$

\footnotetext{
${ }^{a}$ Medical Oncology Unit 2 (Adult Mesenchymal Tumours), Fondazione IRCCS Istituto Nazionale Tumori, Milan, Italy

${ }^{\mathrm{b}}$ Department of Oncology, IRCCS - Istituto di Ricerche Farmacologiche Mario Negri, Milan, Italy

${ }^{\mathrm{c}}$ Molecular Pharmacology Unit, Department of Experimental Oncology and Molecular Medicine, Fondazione IRCCS Istituto Nazionale Tumori, Milan, Italy

${ }^{\mathrm{d}}$ Laboratory of Experimental Molecular Pathology, Department of Diagnostic Pathology and Laboratory, Fondazione IRCCS Istituto Nazionale Tumori, Milan, Italy

${ }^{\mathrm{e}}$ Melanoma and Sarcoma Unit, Department of Surgery, Fondazione IRCCS Istituto Nazionale Tumori, Milan, Italy

${ }^{\mathrm{f}}$ Department of Oncology, University Campus Bio-Medico, via Alvaro del Portillo 21, Rome, Italy

${ }^{\mathrm{g}}$ Department of Surgical, Oncological and Oral Sciences, Section of Medical Oncology, University of Palermo, Palermo, Italy

${ }^{\mathrm{h}}$ Soft Tissue and Bone Pathology, Histopathology and Pediatric Pathology Unit, Department of Diagnostic Pathology and Laboratory Medicine, Fondazione IRCCS Istituto Nazionale dei Tumori, Milan, Italy

${ }^{\mathrm{i}}$ Department of Radiology, Fondazione IRCCS Istituto Nazionale Tumori, Milan, Italy

${ }^{j}$ Department of Anatomic Pathology, General Hospital of Treviso, Treviso, Italy

${ }^{\mathrm{k}}$ Oncology Department, University of Milan, Italy
}

Received 9 November 2016; received in revised form 27 January 2017; accepted 1 February 2017 Available online 8 March 2017

\footnotetext{
Presented at the 20th Connective Tissue Oncology Society (CTOS) annual meeting, Salt Lake City, November 2015, abs \# 039 and at the 52nd American Society of Clinical Oncology (ASCO) annual meeting, Chicago, June 2016, abs \# 11042.

* Corresponding author: Fondazione IRCCS Istituto Nazionale dei Tumori, via Venezian 1, 20133 Milan, Italy. Fax: +39 0223902404. E-mail address: silvia.stacchiotti@istitutotumori.mi.it (S. Stacchiotti). 


\section{KEYWORDS}

Sarcoma;

Solitary fibrous

tumour;

Treatment;

Metastasis;

Chemotherapy;

Anthracycline;

Doxorubicin;

Ifosfamide;

Dacarbazine;

Trabectedin;

Eribulin;

Xenograft;

Mice model
Abstract Background: Preclinical models that mimic pathological and molecular features of solitary fibrous tumour (SFT) represent an important tool to select effective regimes and novel compounds to be tested in the clinic. This study was aimed at developing two preclinical models of SFT, assessing their predictive value in the clinic and selecting potential novel effective treatments.

Material and methods: Two dedifferentiated-SFT (D-SFT) models obtained from patients' biopsies were grown in immunodeficient mice. The antitumour activity on these models of doxorubicin, dacarbazine (DTIC), ifosfamide (monotherapy or combination), trabectedin and eribulin was tested. Twelve SFT patients were treated with doxorubicin and DTIC. Response by RECIST, progression-free survival and overall survival were retrospectively evaluated, distinguishing malignant-SFT (M-SFT) and D-SFT.

Results: Two D-SFT patient-derived xenografts (PDXs) that represent the first available preclinical in vivo models of SFT were developed and characterised. Doxorubicin/DTIC, DTIC/ ifosfamide, doxorubicin/ifosfamide combinations consistently induced better antitumour activity than the single-agents. Particularly, doxorubicin/DTIC combination caused a max tumour volume inhibition $>80 \%$ in both models. Doxorubicin/DTIC combo showed activity also in the case-series. Best RECIST responses were: 6 responses (M-SFT $=2$ of 7, DSFT $=4$ of 5), 1 stable disease, 5 progressions, with a 6 -month median progression-free survival $(\mathrm{M}-\mathrm{SFT}=6, \mathrm{D}-\mathrm{SFT}=10$ months $)$. The PDXs were very sensitive to trabectedin and eribulin.

Conclusion: Doxorubicin plus DTIC combination was effective in our two D-SFT mice models and appeared to be active also in the clinic, especially in high-grade D-SFT patients. Among additional drugs tested in the PDXs, trabectedin and eribulin were highly effective, providing a rational to test these drugs in D-SFT patients.

(c) 2017 Elsevier Ltd. All rights reserved.

\section{Introduction}

Solitary fibrous tumour (SFT) is a rare sarcoma [1,2], marked by a recurrent NAB2-STAT6 gene fusion that is responsible for the nuclear expression of the transcription factor STAT6 [3]. Three clinical-pathologic variants of SFT are identified: typical (T-SFT), malignant (MSFT) and dedifferentiated (D-SFT) SFT $[1,4]$. M-SFT is marked by a greater mitotic index $(\geq 4 / 10$ HPF) compared with T-SFT. D-SFT shows the transition to a high-grade morphology. Notably, STAT6 nuclear immunopositivity can be lost in D-SFT [5]. SFT has a low metastatic potential $(\leq 15 \%)$, but a greater metastatic rate $(40 \%)$ is observed in D-SFT $[6,7]$.

Preliminary evidence is available that both anthracyclines and temozolomide are effective in SFT [8,9]. We had already tested the activity of temozolomide and dacarbazine (DTIC) in a patient-derived xenograft (PDX) model of high-grade SFT, confirming that they looked equally active [10]. In the same paper we reported 8 patients undergoing DTIC, with 3 responses.

The good consistency observed between preclinical and clinical observations encouraged us to develop further this approach. In this study, we report the development and characterisation of two preclinical models of SFT and the results of the experiments aimed at comparing the activity of cytotoxic agents currently used for treatment of soft tissue sarcoma, including the
doxorubicin-DTIC combination, trabectedin and eribulin. We also assessed retrospectively the clinical activity of doxorubicin in combination with DTIC in a small population of advanced SFT patients.

\section{Materials and methods}

\subsection{Experimental models and pharmacological studies}

Two D-SFT PDX models were used in the study.

\subsubsection{Development and characterisation of the model}

The models were established by subcutaneous grafting of tumour fragments obtained at the time of surgery from 2 patients into the right flank of female SCID (SFT-1) or nude (SFT-2) mice (Charles River, Calco, IT). Specifically, SFT-1 was established from a pleomorphic osteochondro-like D-SFT from a patient with recurrent pelvic D-SFT [4]. Both in the patient and in the PDX, the tumour carried the NAB2-STAT6 fusion transcript (ex6.INT6.ex3int), as detected by RT-PCR [3], but lacked immunohistochemical nuclear STAT6 expression [5]. SFT-2 was derived from a patient with locally relapsed D-SFT, pre-treated with sunitinib. This tumour closely resembled an Ewing sarcoma/peripheral primitive neuroectodermal tumour [4]. It was characterised by NAB2-STAT6 fusion transcript (ex6.ex18) and negative STAT6 immunohistochemistry [5]. This 
PDX showed superimposable morphology and, by contrast, recovered STAT6 nuclear expression (Suppl. Fig. 1). Both PDXs showed MGMT methylation [10]. SFT-1 carried a hemizygous and SFT-2 a homozygous $\mathrm{RB} 1$ deletion, as assessed by FISH with BACs mapping on RB1 locus (RP11-305D15, RP11-174I10) together with a control on 13q 31.3 (RP11-121J7) [5]. TP53 was mutated in SFT-2. $17 \mathrm{p}$ deletion, including the TP53 gene, was observed in both clinical tumours by $\mathrm{CGH}$ array [5] (Table 1).

Tumour growth was followed by biweekly measurement of tumour diameters with a Vernier calliper, and tumour volume (TV) calculated by the following formula: TV $\left(\mathrm{mm}^{3}\right)=d^{2} X D / 2$, where $d$ and $D$ are the shortest and the longest diameter, respectively.

The xenograft origin was authenticated through microsatellite analysis [10].

The use of patient material to generate xenografts and all experiments were approved by the institutional Ethics Committees for Animal Experimentation.

\subsubsection{Xenograft treatments}

Treatments started when xenografts were approximately $300 \mathrm{~mm}^{3}$. Eight mice for experimental group were used.
After dilution in sterile water (doxorubicin, DTIC, ifosfamide) or saline solution (trabectedin, eribulin), drugs were administered at dosage-schedules reported in Table 2. Treatment activity was assessed determining TV inhibition percentage $(\mathrm{TVI} \%)$ in treated versus control mice, expressed as $\mathrm{TVI} \%=100-([$ mean $\mathrm{TV}$ treated/mean TV control] $\times 100$ ).

\subsubsection{Immunohistochemical assessment of $\gamma-H 2 A X$ expression}

The immunohistochemical assessment of $\gamma$-H2AX was performed on FFPE PDX samples obtained $24 \mathrm{~h}$ after the first drug treatment and stained with the antiphospho-H2AX (Ser139) mouse monoclonal antibody (Millipore).

\subsubsection{Western blotting}

Lysates from frozen PDX tumours obtained at the end of the last eribulin treatment were prepared [11]. Proteins were separated by SDS-PAGE, transferred onto nitrocellulose membranes and incubated with primary monoclonal antibodies, anti-MPM2, anti-cyclin B1, anti-phospho Cdk1 (Tyr15) and anti- $\beta$-actin [12].

Table 1

Molecular features of patient tumours and corresponding PDX models.

\begin{tabular}{|c|c|c|c|c|c|c|c|}
\hline Sample & Diagnosis & $\begin{array}{l}\text { NAB2-STAT6 } \\
\text { fusion transcript }\end{array}$ & $\begin{array}{l}\text { STAT6 nuclear } \\
\text { immunopositivity }\end{array}$ & $\begin{array}{l}M G M T \text { promoter } \\
\text { methylation }\end{array}$ & $R B 1$ & $\begin{array}{l}\text { TP53 } \\
\text { array CGH [4] }\end{array}$ & $\begin{array}{l}\text { TP53 mutation } \\
\text { analysis }\end{array}$ \\
\hline $\begin{array}{l}\text { Human } \\
\quad \text { sample } 1\end{array}$ & $\begin{array}{l}\text { Pleomorphic } \\
\text { osteochondro-like } \\
\text { dedifferentiated-SFT }\end{array}$ & + & - & + & $\begin{array}{l}\text { Hemyzigous } \\
\text { deletion }\end{array}$ & $\begin{array}{l}\text { Hemyzigous } \\
\text { deletion }\end{array}$ & WT \\
\hline SFT-1 & & + & - & + & $\begin{array}{l}\text { Hemyzigous } \\
\text { deletion }\end{array}$ & nd & WT \\
\hline $\begin{array}{l}\text { Human } \\
\quad \text { sample } 2\end{array}$ & $\begin{array}{l}\text { Ewing-like } \\
\text { dedifferentiated-SFT }\end{array}$ & + & - & + & $\begin{array}{l}\text { Homozygous } \\
\text { deletion }\end{array}$ & $\begin{array}{l}\text { Hemyzigous } \\
\text { deletion }\end{array}$ & G245S \\
\hline SFT-2 & & + & + & + & $\begin{array}{l}\text { Homozygous } \\
\text { deletion }\end{array}$ & nd & $\mathrm{G} 245 \mathrm{~S}$ \\
\hline
\end{tabular}

Abbreviations: SFT, solitary fibrous tumour; nd, not done; WT, wild type.

Table 2

Pharmacological treatments and tumour responses of PDXs treated with doxorubicin, dacarbazine (DTIC), ifosfamide (IFO) and trabectedin, singly administered and in combination.

\begin{tabular}{|c|c|c|c|c|c|}
\hline \multirow[t]{2}{*}{ Treatment } & \multirow[t]{2}{*}{ Dose $(\mathrm{mg} / \mathrm{kg})$} & \multirow[t]{2}{*}{ Schedule } & \multirow[t]{2}{*}{ Route } & \multirow{2}{*}{$\frac{\text { SFT-1 }}{\text { Max TVI } \%{ }^{a}}$} & \multirow{2}{*}{$\frac{\text { SFT-2 }}{\text { Max TVI } \% \text { a }}$} \\
\hline & & & & & \\
\hline Doxorubicin & 2.5 & $q 7 d \times 4$ & i.v. & $27(83)$ & $29(98)$ \\
\hline IFO & 90 & $q d \times 3 q 2 w$ & i.p. & $45(90)$ & $44(98)$ \\
\hline DTIC & 105 & $q 7 d \times 4$ & i.p. & $54(97)$ & $55(94)$ \\
\hline \multirow{2}{*}{ Doxorubicin/DTIC } & 2.5 & $q 7 d \times 4$ & i.v. & $83(90)$ & $96(98)$ \\
\hline & 105 & $q 7 d \times 4$ & i.p. & & \\
\hline \multirow[t]{2}{*}{ IFO/DTIC } & 90 & $\mathrm{qd} \times 3 \mathrm{q} 2 \mathrm{w}$ & i.p. & $74(97)$ & $96(98)$ \\
\hline & 105 & $\mathrm{q} 7 \mathrm{~d} \times 4$ & i.p. & & \\
\hline \multirow[t]{2}{*}{ IFO/doxorubicin } & 90 & $\mathrm{qd} \times 3 \mathrm{q} 2 \mathrm{w}$ & i.p. & $68(97)$ & $93(98)$ \\
\hline & 2.5 & $\mathrm{q} 7 \mathrm{~d} \times 4$ & i.v. & & \\
\hline \multirow[t]{3}{*}{ IFO/doxorubicin/DTIC } & 90 & $\mathrm{qd} \times 3 \mathrm{q} 2 \mathrm{w}$ & i.p. & $97(97)$ & - \\
\hline & 2.5 & $\mathrm{q} 7 \mathrm{~d} \times 4$ & i.v. & & \\
\hline & 105 & $\mathrm{q} 7 \mathrm{~d} \times 4$ & i.p & & \\
\hline Trabectedin & 0.15 & $\mathrm{q} 7 \mathrm{~d} \times 3$ & i.v. & $70(90)$ & $98(103)$ \\
\hline Eribulin & 1 & $\mathrm{q} 4 \mathrm{~d} \times 3 \mathrm{q} 3 \mathrm{w}$ & i.p. & $92(77)$ & - \\
\hline
\end{tabular}

Abbreviations: SFT, solitary fibrous tumour; TVI, tumour volume inhibition; i.v., intravenous; i.p., intraperitoneal.

${ }^{\text {a }}$ Maximum tumour volume inhibition $\%$ in treated versus control mice. In parentheses, the day on which it was assessed. 


\subsection{Patients}

We retrospectively identified 12 patients affected by advanced SFT treated with doxorubicin plus DTIC within 3 centres of the Italian Rare Cancer Network from February 2012 to July 2016. All patients provided a written informed consent to the treatment.

Diagnosis was confirmed by expert sarcoma pathologists, according to the WHO classification [1] and updated criteria [6], reviewing the available tumour sample closest to the start of chemotherapy. STAT6 immunohistochemistry was performed [5]. Seven cases were consistent with M-SFT and 5 with D-SFT.

Table 3 summarises patient characteristics.

Patients received doxorubicin $(75 \mathrm{mg} / \mathrm{mq}$, i.v., bolus) and DTIC ( $800 \mathrm{mg} / \mathrm{mq}$, intravenously over $60 \mathrm{~min}$, in 2 days), every 3 weeks, until a maximum of 6 cycles, unacceptable toxicity or progression. Adverse events were recorded. Disease status was assessed at baseline by a whole body computed tomography scan (CT), a CT or magnetic resonance (MR) of the site(s) of disease, and a whole body bone scan. CT/magnetic resonance scans were repeated every 2 cycles. Response was assessed by RECIST 1.1

Progression-free survival (PFS) and overall survival (OS) were estimated with Kaplan-Meyer method. Failure for PFS was progressive disease by RECIST, or death. Failure for OS was death due to any cause. Patients alive were censored at the time of the last contact.

\section{Results}

\subsection{Experimental model and pharmacological studies}

The antitumour activity of doxorubicin, DTIC and ifosfamide, administered as monotherapy or in combination, was tested against late-stage SFT-1 and SFT-2 xenografts. As monotherapy, DTIC and ifosfamide induced a significant and superimposable tumour growth inhibition in SFT-1 and SFT-2, whereas an almost negligible antitumour activity was observed following doxorubicin treatment in both models (Fig. 1, Table 2). Notably, drugs were delivered at suboptimal doses to highlight the effects of the combined treatments. Doxorubicin/DTIC, DTIC/ifosfamide and doxorubicin/ifosfamide combinations consistently induced an enhanced antitumour activity compared with single-agents, which was mainly appreciable in SFT-2, as indicated by max TVI (mTVI) always $>90 \%$ and long-lasting tumour regressions observed in all mice (Fig. 1, Table 2). Although to a lesser extent, an enhanced therapeutic effect of the two-drug combinations was also observed in the SFT-1, in particular with the doxorubicin/DTIC combination (mTVI $=83 \%$ ). In SFT-1, the three-drug combination (doxorubicin/DTIC/

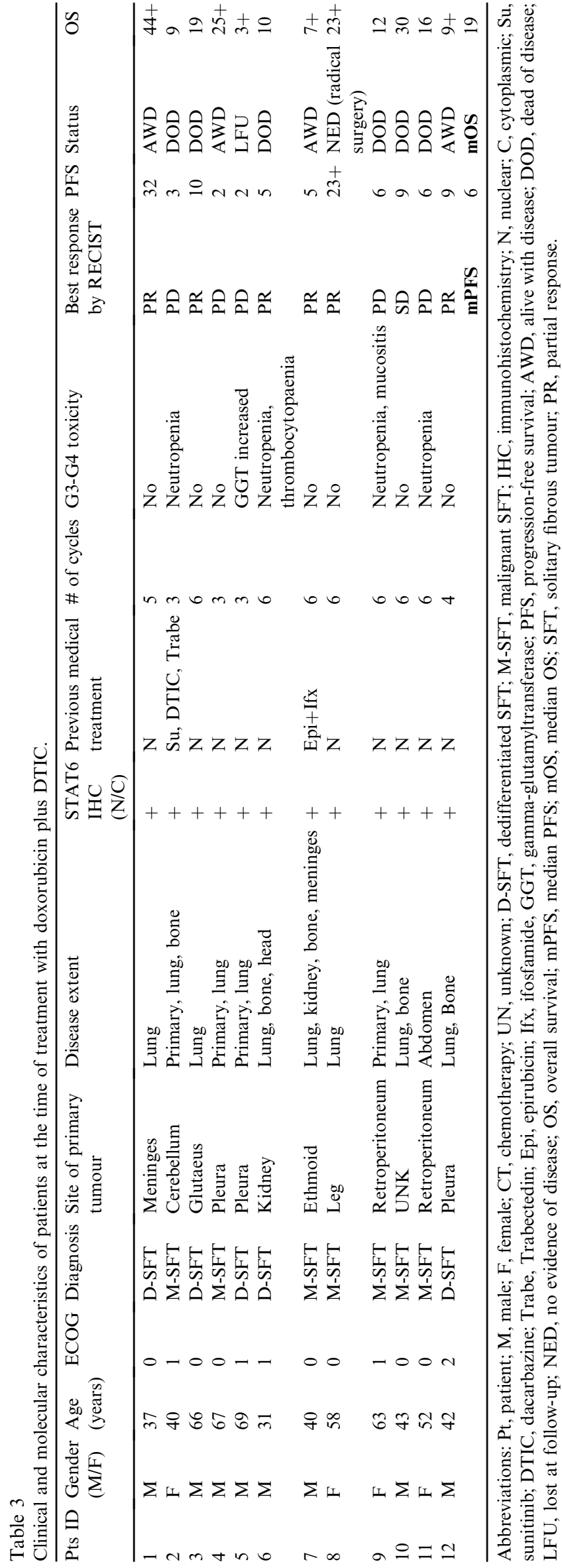



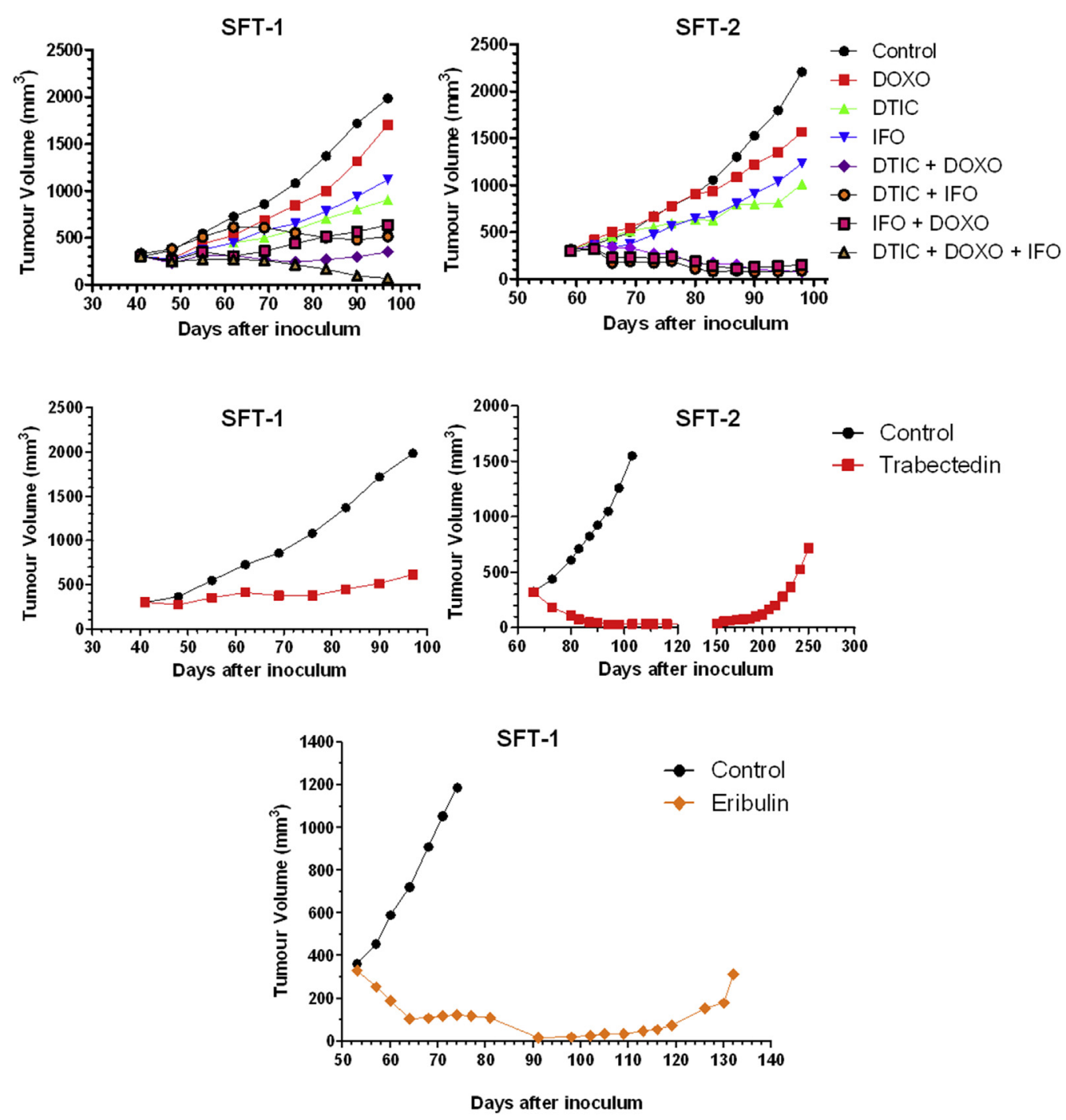

Fig. 1. Antitumour activity of doxorubicin (DOXO), dacarbazine (DTIC), ifosfamide (IFO), trabectedin and eribulin, singly administered and in combination, against solitary fibrous tumours xenotransplanted into SCID (SFT-1) and nude (SFT-2) mice. SFT, solitary fibrous tumour.

ifosfamide) induced the almost complete regression of tumours $(\mathrm{mTVI}=97 \%$; Fig. 1, Table 2$)$.

The expression of $\gamma-\mathrm{H} 2 \mathrm{AX}$, a specific marker for monitoring treatment-induced DNA damage [13], was increased in tumours exposed to the two-drug combinations compared with individual agents in both xenografts (Fig. 2A, Suppl. Fig. 2). A further enhancement was observed in SFT-1 PDXs after treatment with doxorubicin/DTIC/ifosfamide combination (Fig. 2A).

Trabectedin monotherapy induced an mTVI $=70 \%$ in SFT-1, whereas an mTVI $=98 \%$ was detected in SFT-2, which was appreciable until two months from the end of treatment (Fig. 1, Table 2). $\gamma$-H2AX expression levels indicated that trabectedin caused less DNA damage (Fig. 2A, Suppl. Fig. 2) than doxorubicin/DTIC, DTIC/ifosfamide and doxorubicin/ifosfamide combinations, consistent with the notion that trabectedin activity is mainly related to the effect on transcription regulation [14].
Eribulin activity, tested only against SFT-1, induced an $\mathrm{mTVI}=92 \%$, with 4 mice experiencing long-lasting complete responses, 2 of which were maintained until the end of the experiment (Fig. 1).

Biochemical analysis on eribulin-treated xenografts showed increased staining of MPM-2 antibody, which recognises mitotic phosphoproteins [15], together with enhanced cyclin B1 levels and dephosphorylation on inhibitory Tyr-15 of Cdk1 [16], consistent with a tumour cell accumulation in the G2/M phase (Fig. 2B).

\subsection{Patients}

Twelve patients with advanced SFT received doxorubicin combined with DTIC. Mean number of cycles was 5. The treatment was well tolerated and toxicity was as expected (Table 3 ).

All patients were assessable for response. The best RECIST response was partial response (PR) in $6(50 \%)$, 
A

B
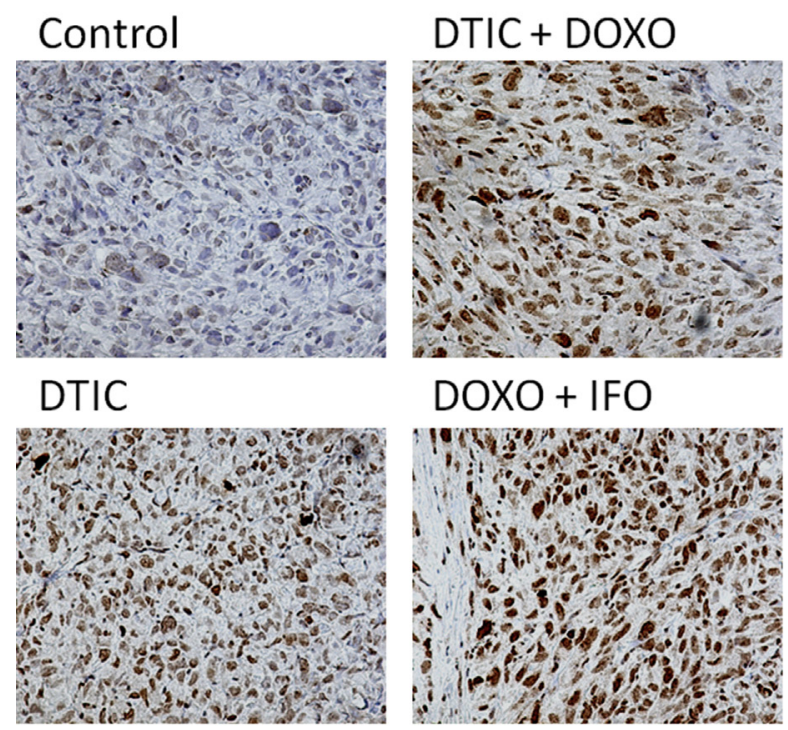

$\mathrm{DOXO}+\mathrm{IFO}$
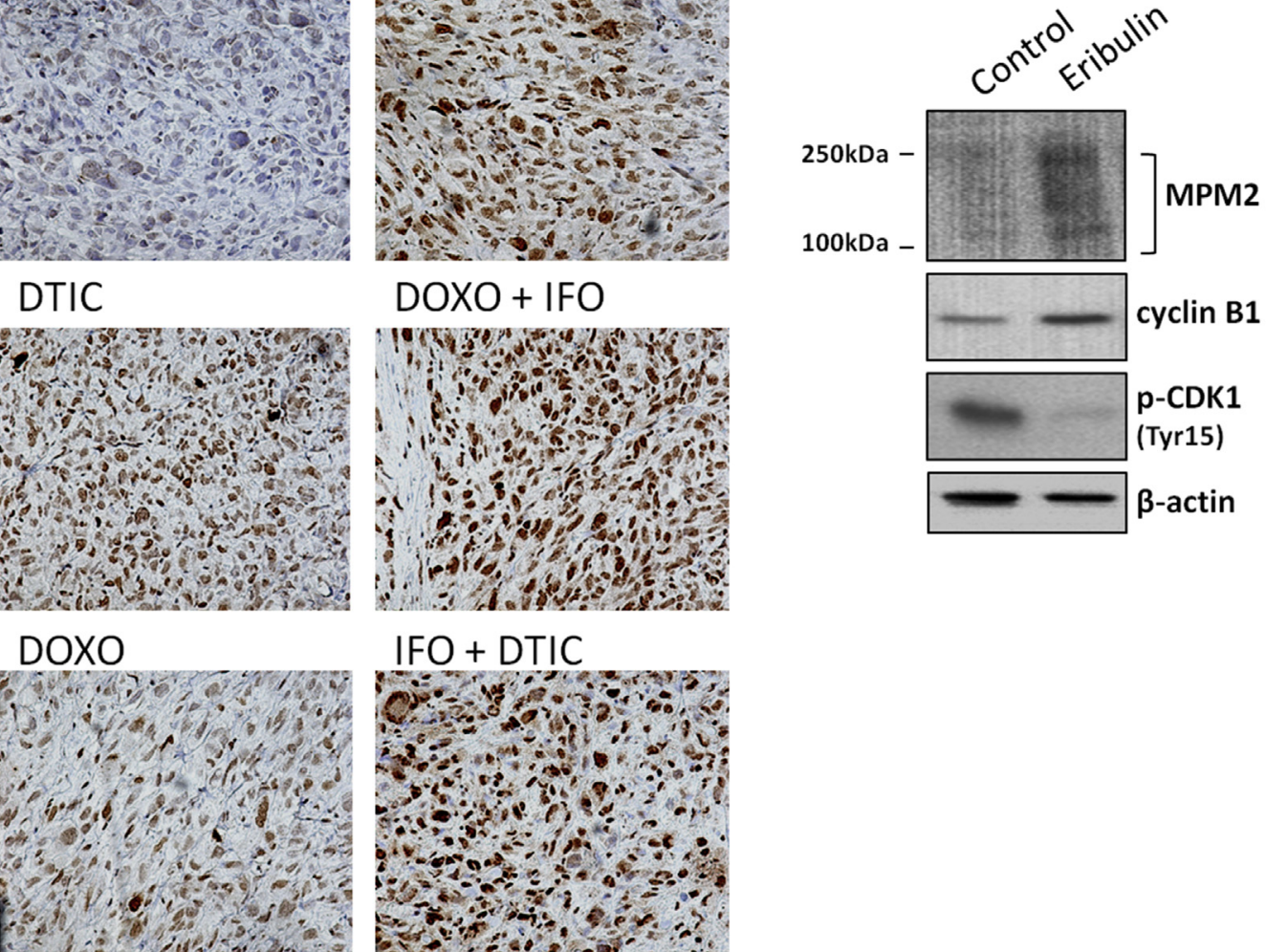

IFO + DTIC
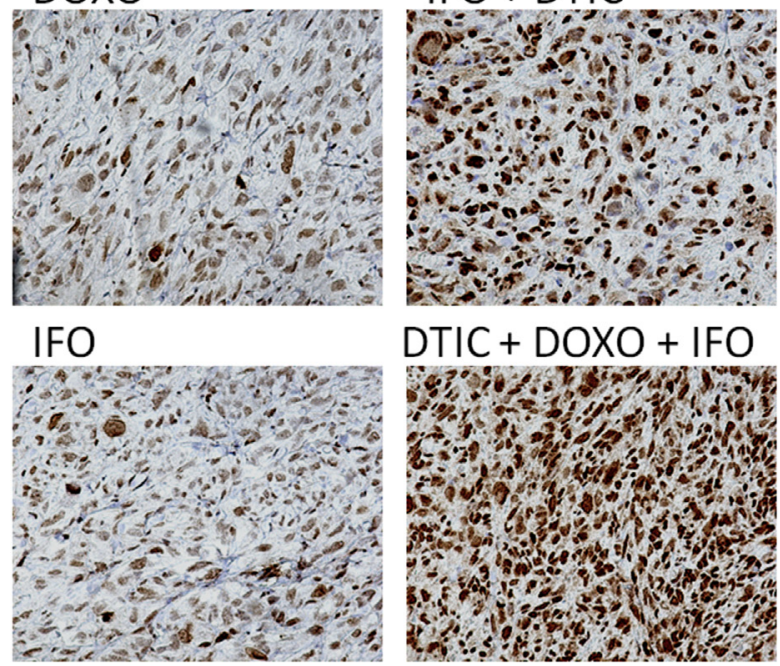

$D T I C+D O X O+I F O$

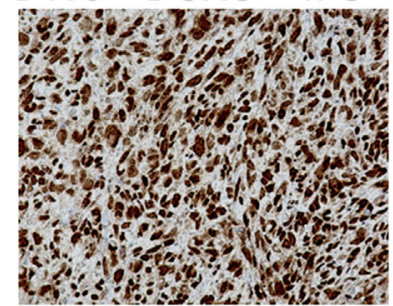

Trabectedin

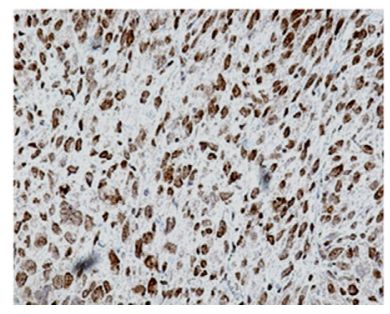

Fig. 2. A) $\gamma-\mathrm{H} 2 \mathrm{AX}$ nuclear immunostaining in SFT-1 tumours following exposure to doxorubicin (DOXO), dacarbazine (DTIC), ifosfamide (IFO) and trabectedin, singly administered and in combination. B) Western blot analysis of mitosis-related factors in SFT-tumour following exposure to eribulin. SFT, solitary fibrous tumour.

stable disease in $1(8.3 \%)$ and progression in $5(41.7 \%)$ cases (Table 3, Fig. 3). A RECIST PR was detected in $2 /$ $7 \mathrm{M}$-SFT and in 4/5 D-SFT.

At a 25-month median follow-up, the median OS was 19 months (range 9-44+), with 7 patients dead at the time of the analysis. The median PFS was 6 months (range 2-32), with $20 \%$ of patients progression-free at
12 months. Median PFS was 6 and 10 in M-SFT and DSFT, respectively $(\mathrm{p}=0.37)$.

\section{Discussion}

No prospective studies focussing on chemotherapy are available in SFT. Of course, their rarity makes 

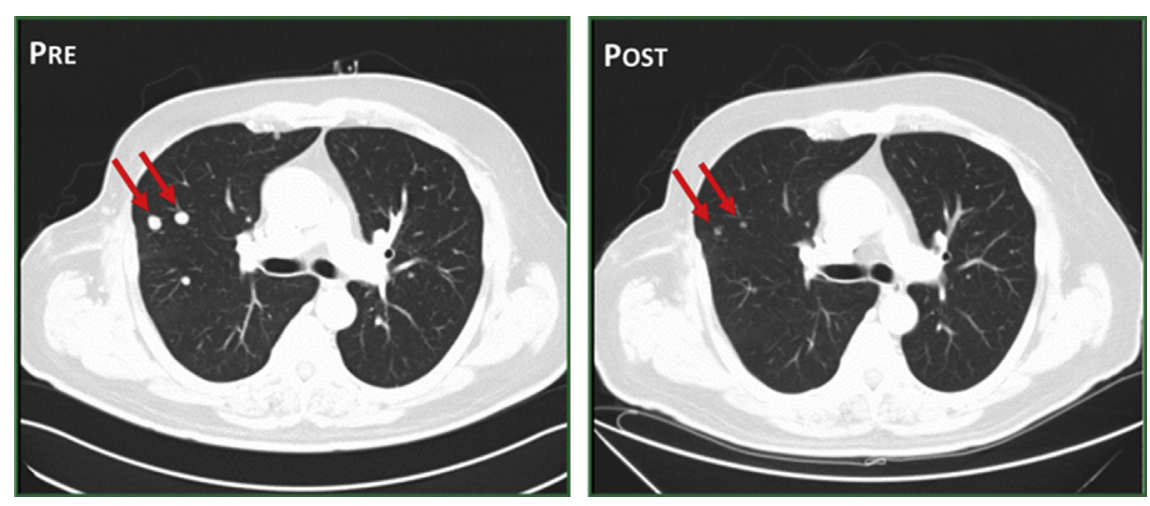

Fig. 3. Axial, contrast enhanced CT scans in a man affected by lung metastases (red arrows) from a dedifferentiated solitary fibrous tumour of the glutaeus, at baseline (left panel) and after 6 cycles of treatment with doxorubicin and DTIC (right panel). The lung lesions in the middle lobe reduced from 12 to $10 \mathrm{~mm}$ to 6 and $5 \mathrm{~mm}$, respectively, thus achieving a partial response according to RECIST. SFT, solitary fibrous tumour; DTIC, dacarbazine. (For interpretation of the references to colour in this figure legend, the reader is referred to the web version of this article.)

comparative assessments in clinical studies challenging. Thus, retrospective series and predictive preclinical models are important to select treatments to be prospectively tested in clinical trials. PDX models, obtained through the direct implant in immunodeficient mice of surgical tumour specimens, are particularly promising, since they retain the main molecular, genetic and histologic features of donor tumours, closely recapitulating the original heterogeneity [17]. An advantage of PDXs compared with cell-lined-derived xenografts is their ability to better predict the response to therapeutic agents and to provide the insights useful for drug scheduling [18].

We already reported the consistency between preclinical and clinical results concerning the activity of temozolomide and DTIC [10] as well as that of several antiangiogenics [19] in our first D-SFT model (SFT-1). These results prompted us to develop further models and to compare the activity of the cytotoxic drugs available for treatment of sarcomas.

By assessing the activity of doxorubicin, ifosfamide and DTIC (as monotherapy or in combination) in two D-SFT PDXs, we observed that all combinations consistently induced an increased antitumuor activity compared with single-agents. Such an enhancement was mainly appreciable with the doxorubicin/DTIC combination (mTVIs $=83 \%$ and $96 \%$ observed in SFT- 1 and SFT-2, respectively). Interestingly, the three-drug combination doxorubicin/DTIC/ifosfamide induced an almost complete regression of SFT-1 xenograft tumours. The increased antitumour activity was parallelled by an enhanced $\gamma \mathrm{H} 2 \mathrm{AX}$ staining in tumours, thus indicating the cumulative induction of lethal DNA lesions as a main mechanism responsible for the therapeutic efficacy of the combinations.

It is noteworthy that in these preclinical experiments suboptimal doses of individual agents were used to better appreciate the effect of combos. However, a remarkable therapeutic effect was also appreciable when higher doses of DTIC [10] or doxorubicin (Suppl. Fig. 3) were used as single-agents. This may be relevant for less fit and/or elder patients when a disease stabilisation is acceptable and a monotherapy with an expected better toxicity profile can be the choice.

Available data on the role of chemotherapy in patients with SFT are limited and point to the activity of agents like anthracycline, DTIC, ifosfamide $[20,21]$ and trabectedin $[22,23]$. Two responses to eribulin were also reported $[24,25]$. The expected $\mathrm{RR}$ is low both with anthracycline-based regimens $(0-20 \%)$ and with trabectedin (9\%) [8,21,23,24,26-28]. In 2013, we published a retrospective series of 31 SFT patients treated with anthracycline-based chemotherapy (anthracycline monotherapy: 8; anthracycline/ifosfamide: 23 ). We observed $20 \%$ RECIST PR and a 4-month median PFS [8]. Notably, PRs were found in 2/18 (11\%) M-SFT e 4/12 $(30 \%)$ D-SFT, with a median PFS of 3.5 and 5 months in M-SFT and D-SFT, respectively. The finding that the doxorubicin/DTIC combination was active in our DSFT models prompted us to assess the efficacy of this combination in the clinic. The case-series analysis that we could collect and present herein, with all the limitations of a small and retrospective series of patients, confirms that anthracycline-based regimens are effective in SFT, showing that the combination of doxorubicin/ DTIC seems to increase both the RR (50\% PR by RECIST) and PFS (6-month median PFS) compared with our prior series. As already observed [8], D-SFT appears more sensitive to chemotherapy compared with M-SFT, both in terms of RR and of PFS (10 versus 6 months). This suggests preferring a combination of doxorubicin/DTIC in high-grade cases and when a major tumour response is needed.

In the D-SFT models, we also tested the activity of other drugs of clinical interest for sarcomas, such as trabectedin and eribulin.

Trabectedin showed remarkable antitumour activity in both models. Indeed, a major (SFT-1) or almost 
complete (SFT-2) tumour regression was found, in contrast with clinical data that are more consistent with a disease stabilisation without tumour shrinkage [22]. Since clinical reports do not detail which is the SFT subtype of those patients who benefited from trabectedin, the possibility that SFT biologic aggressiveness is responsible for a different sensitivity to the drug cannot be ruled out.

An impressive antitumour activity, with long-lasting complete tumour regressions, was also observed in SFT-1 following treatment with the novel microtubule inhibitor eribulin [29]. Consistently with its antimitotic effect, biochemical analysis on eribulin-treated xenografts was suggestive of tumour cell accumulation in the G2/M phase. Eribulin was recently approved by FDA and EMA for treatment of advanced liposarcoma resistant to anthracycline, based on the results of a phase 3 trial [30]. In two phase 2 studies that were run in soft tissue sarcoma, two anecdotal responses were detected in a SFT patients $[24,25]$. This preliminary clinical observation, together with the impressive preclinical results, suggests evaluating further eribulin in SFT.

In conclusion, our results provide insights into the activity of cytotoxic agents available in the clinical practice for treatment of STS, confirming that, especially in the more aggressive SFT, they actually belong to the therapeutic medical armamentarium. Based on these results, a prospective phase 2 randomised study on doxorubicin/DTIC versus trabectedin in advanced SFT is starting. In addition, eribulin, the last cytotoxic agent approved for the treatment of sarcoma, deserves further investigations. Finally, additional experiments are ongoing in our PDXs to investigate the efficacy of new molecular targeted agent as single-agents and in combination with cytotoxics in the disease.

\section{Conflict of interest statement}

Stacchiotti S, Gronchi A, Casali PG, D'Incalci M: Pharmamar: compensated advisory board, honoraria, research funding; Vincenzi B, Badalamenti G, Casali PG: EISAI: compensated advisory board, honoraria, research funding; and the remaining authors have no conflicts to declare.

\section{Appendix A. Supplementary data}

Supplementary data related to this article can be found at http://dx.doi.org/10.1016/j.ejca.2017.02.002.

\section{References}

[1] Fletcher CDM, Bridge JA, Hogendoorn P, Mertens F. World Health Organization (WHO) classification of tumours of soft tissue and bone. Pathology and genetics. Lyon: IARC Press; 2013. p. $328-9$.
[2] Verbeke SLJ, Fletcher CD, Alberghini M, Daugaard S, Flanagan AM, Parratt T, et al. A reappraisal of hemangiopericytoma of bone; analysis of cases reclassified as synovial sarcoma and solitary fibrous tumor of bone. Am J Surg Pathol 2010;34:777-83.

[3] Robinson DR, Wu YM, Kalyana-Sundaram S, Cao X, Lonigro RJ, Sung YS, et al. Identification of recurrent NAB2STAT6 gene fusions in solitary fibrous tumor by integrative sequencing. Nat Genet 2013;45:180-5.

[4] Collini P, Negri T, Barisella M, Palassini E, Tarantino E, Pastorino U, et al. High-grade sarcomatous overgrowth in solitary fibrous tumors: a clinicopathologic study of 10 cases. Am J Surg Pathol 2012;36:1202-15.

[5] Dagrada GP, Spagnuolo RD, Mauro V, Tamborini E, Cesana L, Gronchi A, et al. Solitary fibrous tumors: loss of chimeric protein expression and genomic instability mark dedifferentiation. Mod Pathol 2015;28:1074-83.

[6] Mosquera JM, Fletcher CDM. Expanding the spectrum of malignant progression in solitary fibrous tumors: a study of 8 cases with a discrete anaplastic component-is this dedifferentiated SFT? Am J Surg Pathol 2009;33:1314-21.

[7] Magro G, Emmanuele C, Lopes M, Vallone G, Greco P. Solitary fibrous tumour of the kidney with sarcomatous overgrowth. Case report and review of the literature. APMIS 2008;116:1020-5.

[8] Stacchiotti S, Libertini M, Negri T, Palassini E, Gronchi A, Fatigoni S, et al. Response to chemotherapy of solitary fibrous tumour: a retrospective study. Eur J Cancer 2013;49:2376-83.

[9] Park MS, Patel SR, Ludwig JA, Trent JC, Conrad CA, Lazar AJ, et al. Activity of temozolomide and bevacizumab in the treatment of locally advanced, recurrent, and metastatic hemangiopericytoma and malignant solitary fibrous tumor. Cancer 2011;117:4939-47.

[10] Stacchiotti S, Tortoreto M, Bozzi F, Tamborini E, Morosi C, Messina A, et al. Dacarbazine in solitary fibrous tumor: a case series analysis and preclinical evidence vis-a-vis temozolomide and antiangiogenics. Clin Cancer Res 2013;19:5192-201.

[11] De Cesare M, Lauricella C, Veronese S, Cominetti D, Pisano C, Zunino F, et al. Synergistic antitumor activity of cetuximab and namitecan in human squamous cell carcinoma models relies on cooperative inhibition of EGFR expression and depends on high EGFR gene copy number. Clin Cancer Res 2014;20:995-1006.

[12] Zuco V, Zunino F. Cyclic pifithrin-alpha sensitizes wild type p53 tumor cells to antimicrotubule agent-induced apoptosis. Neoplasia 2008;10:587-96.

[13] Firsanov DV, Solovjeva LV, Svetlova MP. H2AX phosphorylation at the sites of DNA double-strand breaks in cultivated mammalian cells and tissues. Clin Epigenetics 2011;2:283-97.

[14] Larsen AK, Galmarini CM, D'Incalci M. Unique features of trabectedin mechanism of action. Cancer Chemother Pharmacol 2016;77:663-71.

[15] Tapia C, Kutzner H, Mentzel T, Savic S, Baumhoer D, Glatz K. Two mitosis-specific antibodies, MPM-2 and phospho-histone H3 (Ser28), allow rapid and precise determination of mitotic activity. Am J Surg Pathol 2006;30:83-9.

[16] Timofeev O, Cizmecioglu O, Settele F, Kempf T, Hoffmann I. Cdc25 phosphatases are required for timely assembly of CDK1cyclin B at the G2/M transition. J Biol Chem 2010;285:16978-90.

[17] Cassidy JW, Caldas C, Bruna A. Maintaining tumor heterogeneity in patient-derived tumor xenografts. Cancer Res 2015;75: 2963-8.

[18] Rosfjord E, Lucas J, Li G, Gerber HP. Advances in patient-derived tumor xenografts: from target identification to predicting clinical response rates in oncology. Biochem Pharmacol 2014;91:135-43.

[19] Stacchiotti S, Tortoreto M, Baldi GG, Grignani G, Toss A, Badalamenti G, et al. Preclinical and clinical evidence of activity of pazopanib in solitary fibrous tumor. Eur J Cancer 2014;50: 3021-8.

[20] Chamberlain MC, Glantz MJ. Sequential salvage chemotherapy for recurrent intracranial hemangiopericytoma. Neurosurgery 2008;63:720-6. 
[21] Park MS, Ravi V, Conley A, Patel SR, Trent JC, Lev DC, et al. The role of chemotherapy in advanced solitary fibrous tumors: a retrospective analysis. Clin Sarcoma Res 2013;3:7.

[22] Chaigneau L, Kalbacher E, Thiery-Vuillemin A, FagnoniLegat C, Isambert $\mathrm{N}$, Aherfi L, et al. Efficacy of trabectedin in metastatic solitary fibrous tumor. Rare Tumors 2011;3:e29.

[23] Khalifa J, Ouali M, Chaltiel L, Le Guellec S, Le Cesne A, Blay JY, et al. Efficacy of trabectedin in malignant solitary fibrous tumors: a retrospective analysis from the French Sarcoma Group. BMC Cancer 2015;15:700.

[24] Schöffski P, Ray-Coquard IL, Cioffi A, Bui NB, Bauer S, Hartmann JT, et al. Activity of eribulin mesylate in patients with soft-tissue sarcoma: a phase 2 study in four independent histological subtypes. Lancet Oncol 2011;12:1045-52.

[25] Kawai A, Araki N, Naito Y, Ozaki T, Sugiura H, Yazawa Y, et al. Phase 2 study of eribulin in patients with previously treated advanced or metastatic soft tissue sarcoma. Jpn J Clin Oncol 2017:47:137-44.
[26] Galanis E, Buckner JC, Scheithauer BW, Kimmel DW, Schomberg PJ, Piepgras DG. Management of recurrent meningeal hemangiopericytoma. Cancer 1998;82:1915-20.

[27] Ferigo N, Cottalorda J, Allard D, Gentil-Perret A, Fessy M, Berger C, et al. Successful treatment via chemotherapy and surgical resection of a femoral hemangiopericytoma with pulmonary metastasis. J Pediatr Hematol Oncol 2006;28:237-40.

[28] Beadle GF, Hillcoat BL. Treatment of advanced malignant hemangiopericytoma with combination adriamycin and DTIC: a report of four cases. J Surg Oncol 1983;22:167-70.

[29] Dybdal-Hargreaves NF, Risinger AL, Mooberry SL. Eribulin mesylate: mechanism of action of a unique microtubule-targeting agent. Clin Cancer Res 2015;21:2445-52.

[30] Schöffski P, Chawla S, Maki RG, Italiano A, Gelderblom H, Choy E, et al. Eribulin versus dacarbazine in previously treated patients with advanced liposarcoma or leiomyosarcoma: a randomised, open-label, multicentre, phase 3 trial. Lancet 2016;387: $1629-37$. 